\title{
Saliency and Optical Flow for Gaze Guidance in Videos
}

\author{
Srinivas Sridharan* \\ Reynold Bailey \\ Rochester Institute of Technology
}

\begin{abstract}
Computer-based gaze guidance techniques have important applications in computer graphics, data visualization, image analysis, and training. Bailey et al. [2009] showed that it is possible to influence exactly where attention is allocated using a technique called Subtle Gaze Direction (SGD). The SGD approach combines eye tracking with brief image-space modulations in the peripheral regions of the field of view to guide viewer gaze about a scene. A fast eye-tracker is used to monitor gaze in real-time and the modulations are terminated before they can be scrutinized by the viewer's high acuity foveal vision. The SGD technique has been shown to improve spatial learning, visual search task performance, and problem solving in static digital imagery [Sridharan et al. 2012]. However, guiding attention in videos is challenging due to competing motion cues in the visual stimuli. We propose a novel method that uses scene saliency (spatial information) and optical flow (temporal information) to enable gaze guidance in dynamic scenes. The results of a user study show that the accuracy of responses to questions related to target regions in videos was higher among subjects who were gaze guided with our approach compared to a control group that was not actively guided.
\end{abstract}

Keywords: gaze manipulation, eye-tracking, optical flow, saliency Concepts: •Computing methodologies $\rightarrow$ Image manipulation; Image processing;

\section{Introduction}

Humans rely heavily on visual information from the environment to perform everyday tasks. When viewing static images, the viewer's gaze pattern is guided by a variety of influences (bottom-up and topdown). We present a novel framework for gaze guidance on videos using visual scene saliency from each video frame and the optical flow information from a series of frames to modulate spatial regions to actively guide viewer attention. We conducted a user study to analyze the effectiveness of guiding viewer gaze about video stimuli and compare their performance to viewers in an unguided control group.

\section{System Design}

Our gaze guidance system uses scene saliency and optical flow information to actively guide viewer attention to different desired regions of interest (ROIs) in video frames (see Figure 1). The target regions are manually selected by the researchers beforehand. Saliency is computed using low-level, mid-level, and highlevel visual features to predict likely regions of interest that the

*e-mail:sxs9716@rit.edu

Permission to make digital or hard copies of part or all of this work for personal or classroom use is granted without fee provided that copies are not made or distributed for profit or commercial advantage and that copies bear this notice and the full citation on the first page. Copyrights for thirdparty components of this work must be honored. For all other uses, contact the owner/author(s). (c) 2016 Copyright held by the owner/author(s).

SAP '16, July 22-23, 2016, Anaheim, CA, USA

ISBN: 978-1-4503-4383-1/16/07

DOI: http://dx.doi.org/10.1145/2931002.2948725

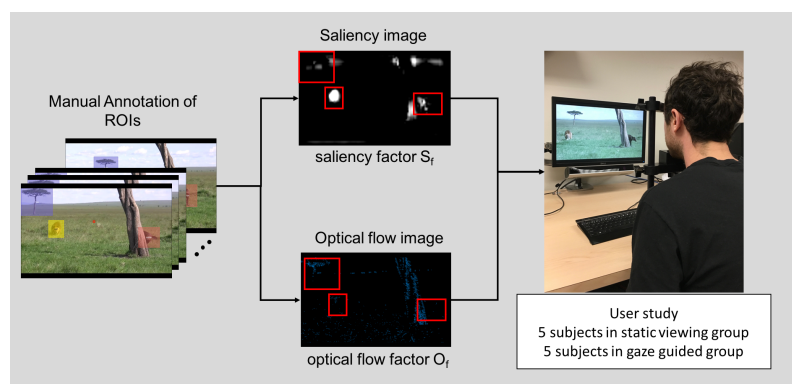

Figure 1: Sample video with ROIs annotated manually. saliency and optical flow factors are extracted for each ROI (highlighted using red box) for gaze guidance.

viewer would look at [Judd et al. 2009] and optical flow vectors are computed by estimating motion between consecutive video frames [Farneback 2003]. Our system uses a GPU and a multithreaded CPU approach to change the intensity of modulations based on the targets region's saliency factor $\left(S_{f}\right)$, and the transparency of modulations based on the targets region's optical flow factor $\left(O_{f}\right)$.

\section{Results}

We conducted a pilot study using 10 videos and 10 subjects ( 5 subjects gaze guided, 5 subjects in control group). We measured the accuracy of the responses from the gaze guided group and the control group to questions related to the target regions in the video. Subjects in the gaze guided group had an accuracy of $82 \%$ compared to an accuracy of $48 \%$ for the static viewing group. This work has application in online learning, gaming and advertisement.

\section{Acknowledgements}

This material is based on work supported be the National Science Foundation under Award No. IIS-0952631. Any opinions, findings, and conclusions or recommendations expressed in this material are those of the author(s) and do not necessarily reflect the views of the National Science Foundation.

\section{References}

Bailey, R., McNAmara, A., Sudarsanam, N., AND GRImm, C. 2009. Subtle gaze direction. ACM Trans. Graph. 28, 4 (Sept.), 100:1-100:14.

FARnebACK, G. 2003. Two-frame motion estimation based on polynomial expansion. In Image analysis. Springer, 363-370.

Judd, T., Ehinger, K., Durand, F., And Torralba, A. 2009. Learning to predict where humans look. In IEEE International Conference on Computer Vision (ICCV).

Sridharan, S., Bailey, R., McNamara, A., And Grimm, C. 2012. Subtle gaze manipulation for improved mammography training. In Proceedings of the Symposium on Eye Tracking Research and Applications, ACM, 75-82. 